\title{
Higher plasma NT-proBNP levels correlate with syndrome of inappropriate antidiuretic hormone and poor prognosis in neurological patients
}

\author{
Qing-Ling Zeng ${ }^{1,2}$, Wen-Tao $\mathrm{He}^{2}$, Gang Yuan ${ }^{2}$ \\ ${ }^{1}$ Merck Serono (China) Co. Ltd., Chengdu, China; ${ }^{2}$ Department of Endocrinology, Tongji Hospital, Tongji Medical College, Huazhong University \\ of Science and Technology, Wuhan, China \\ Contributions: (I) Conception and design: WT He, G Yuan; (II) Administrative support: G Yuan; (III) Provision of study materials or patients: G Yuan; \\ (IV) Collection and assembly of data: QL Zeng; (V) Data analysis and interpretation: QL Zeng, WT He; (VI) Manuscript writing: All authors; (VII) \\ Final approval of manuscript: All authors. \\ Correspondence to: Wen-Tao He; Gang Yuan. Department of Endocrinology, Tongji Hospital, Tongji Medical College, Huazhong University of \\ Science and Technology, Branch of National Clinical Research Center for Metabolic Disease, 1095 Jiefang Road, Wuhan 430030, China. Email: \\ wthe@tjh.tjmu.edu.cn; gangyuan@tjh.tjmu.edu.cn.
}

Background: Hyponatremia induced by syndrome of inappropriate antidiuretic hormone secretion (SIADH) was common electrolyte disturbance encountered in critically ill neurological diseases, which has normal or increased fluid volume. Brain natriuretic peptide (BNP), which is released in equal proportion to N-terminal pro-brain natriuretic peptide (NT-proBNP), plays vital roles in regulation of volume status. The relationship between SIADH and NT-proBNP levels in neurological diseases has rarely been reported.

Methods: A retrospective cross-sectional study was conducted to analyze plasma NT-proBNP levels in 33 patients with SIADH and 23 controlled eunatremic patients with neurological diseases.

Results: Baseline NT-proBNP levels were compared between two groups [SIADH group: median $311 \mathrm{pg} / \mathrm{mL}$, interquartile range (IQR) $110-768 \mathrm{pg} / \mathrm{mL}$ ] vs. eunatremic group: median $46 \mathrm{pg} / \mathrm{mL}, \mathrm{IQR}, 12$ $96 \mathrm{pg} / \mathrm{mL})(\mathrm{P}<0.05)$. Plasma NT-proBNP levels were markedly increased in hyponatremic patients who had two or more complications than those who had less complication $(\mathrm{P}<0.05)$. In SIADH patients, NT-proBNP levels in remission phase were lower to levels at baseline. Furthermore, no death was seen in eunatremic patients, while five SIADH patients died from complications.

Conclusions: SIADH had higher plasma NT-proBNP levels and poorer prognosis compared to eunatremic neurological patients. NT-proBNP serves as a biomarker of disease severity while not extracellular volume (ECV) status in critically ill neurological patients.

Keywords: N-terminal pro-brain natriuretic peptide (NT-proBNP); hyponatremia; syndrome of inappropriate antidiuretic hormone (SIADH)

Submitted Apr 18, 2020. Accepted for publication Sep 25, 2020.

doi: $10.21037 / \mathrm{atm}-20-3413$

View this article at: http://dx.doi.org/10.21037/atm-20-3413 


\section{Introduction}

Hyponatremia is the commonest electrolyte disorder in hospitalized patients, especially in neurological patients $(1,2)$. The majority of researches define hyponatremia as serum concentration of sodium less than $135 \mathrm{mmol} / \mathrm{L}$. Several large-scale epidemiologic studies showed that the prevalence of hyponatremia ranged from $17.5 \%$ to $22.1 \%$ in hospitalized patients (3-5). Other groups reported relatively lower prevalence of hyponatremia which was around $4.5 \%$ depending on the underlying conditions $(6,7)$. Nevertheless, it has been widely recognized that up to 10 $50 \%$ of neurological patients have concurrent hyponatremia $(8-10)$. Fluctuation in the plasma osmolarity is detected by specialized neurons, namely osmoreceptors, located in the anteromedial hypothalamus. It appears that decreased plasma sodium or osmolarity under specific set point triggers the secretion of arginine vasopressin (AVP) to keep stable plasma sodium or osmolarity. In neurological patients, hyponatremia is largely related to excessive production of AVP despite reduced plasma osmolarity, well known as syndrome of inappropriate antidiuretic hormone secretion (SIADH). AVP exerts antidiuretic effect through enhanced reabsorption of solute-free water in the dismal tubules and collecting ducts. Therefore, patients with SIADH have normal or increased extracellular volume (ECV) (11). The resultant extracellular hypo-osmolarity in acute SIADH causes increased intracranial pressure (ICP) and brain edema. So, acute encephalopathy mediated by SIADH is a life-threatening emergency. In a retrospective study, data showed that the majority of patients with hyponatremia who died are caused by progressive underlying illnesses but not by neurologic complications from hyponatremia (12). So, hyponatremia or SIADH seems to serve as an important marker for disease severity in critically ill patients $(13,14)$.

Natriuretic peptide family members are supposed to play indispensable roles to counteract expansion of ECV mediated by AVP. Brain natriuretic peptide (BNP) is widely known as fluid homeostasis regulator secreted by myocardiocytes in response to volume expansion and pressure overload $(15,16)$. In healthy volunteers, it has been demonstrated that elevation of plasma BNP concentration by $44 \%$ is paralleled with increased renal sodium excretion by $60 \%$ (17). BNP and its N-terminal counterpart, $\mathrm{N}$-terminal pro-BNP (NT-proBNP), are cardiac biomarkers that have been established for the assessment of congestive heart failure (16). In patients with $\mathrm{SIADH}$, they tend to have more severe underlying diseases and increased stress responses. The blood pressure, heart rates and pro-inflammatory cytokines are presumably increased in these patients. As we know, cardiovascular overload and uncontrolled inflammation are contributing factors to increase BNP levels (18). BNP, which was initially isolated from extracts of porcine brain (19), had also been found to be mainly expressed in hypothalamus using different primate models (20). Moreover, neurons responsible for AVP secretion are also located in the same area with BNP-secreting neurons. It remains open whether aberrant secretion of AVP correlates with BNP release in neurological patients with SIADH. It has long been demonstrated that poststroke patients with higher BNP levels have increased mortality rate (21). In other neurological diseases, such as traumatic brain injury and subarachnoid hemorrhage (SAH), it has also been demonstrated that BNP levels positively correlated with worse prognosis $(22,23)$.

SIADH is the commonest hyponatremia in neurological patients. As mentioned above, SIADH often reflects the severity of underlying diseases which have contributing factors to increase BNP levels in neurological patients, such as cardiovascular overload, aberrant inflammation and brain damage. In addition, secretion of BNP responds to expansion of fluid volume which is mediated by excessive absorption of free water, so as to mediate sodium excretion in the urine. Theoretically, BNP levels have a potential link with SIADH and prognosis in these patients. To date, limited work has been performed on the relationship between NT-proBNP levels and SIADH in neurological patients. Therefore, we conducted a single-center retrospective study to address this issue. We present the following article in accordance with the reporting checklist (available at http://dx.doi.org/10.21037/atm-20-3413).

\section{Methods}

Written informed consent was obtained from all participating patients on admission. This study was approved by the ethics committee of Tongji Hospital, Tongji Medical College, Huazhong University of Science and Technology (Institutional Review Board Approval No. TJ-C20190101). All procedures were carried out in accordance with the principles of the Declaration of Helsinki (as revised in 2013). We conducted a cross-sectional study to compare the NT-proBNP levels in neurological patients with or without SIADH in the department of neurology of our hospital between June 30, 2012 and May 31, 2016. Hyponatremia 
was defined as concentration of plasma sodium $\leq 135 \mathrm{mmol} / \mathrm{L}$. The underlying diseases include SIADH and eunatremia from patients with intracranial diseases. These diseases encompass cerebral ischemic stroke, cerebral or SAH, viral or tubercular meningitis and intracranial infection. Patients who had cardiovascular, nephrological or other comorbidities markedly affected plasma NT-proBNP levels should be excluded. The patients on admission who had severe heart dysfunction, renal insufficiency, nephrotic syndrome, hypothyroidism, adrenocortical insufficiency, liver cirrhosis or multiple organ failure were excluded from the study. In hyponatremic group, 33 patients were diagnosed as SIADH according to diagnostic criteria adapted from European standard published previously (24), which contained essential criteria and supplemental criteria. The essential criteria included (I) effective serum osmolality <275 $\mathrm{mOsm} / \mathrm{kg} \cdot \mathrm{H}_{2} \mathrm{O}$, (II) urine osmolality $>100 \mathrm{mOsm} / \mathrm{kg} \cdot \mathrm{H}_{2} \mathrm{O}$ at some level of decreased effective osmolality, (III) clinical euvolemia, (IV) urine sodium concentration $>30 \mathrm{mmol} / \mathrm{L}$ with normal dietary salt and water intake, (V) absence of adrenal, thyroid, pituitary or renal insufficiency, and (VI) no recent use of diuretic agents. The supplemental criteria contain (I) serum uric acid $<0.24 \mathrm{mmol} / \mathrm{L}$ ( $<4 \mathrm{mg} / \mathrm{dL})$, (II) serum urea $<3.6 \mathrm{mmol} / \mathrm{L}$ (<21.6 mg/dL), (III) failure to correct hyponatremia after $0.9 \%$ saline infusion, (IV) fractional sodium excretion $>0.5 \%$, (V) fractional urea excretion $>55 \%$, (VI) fractional uric acid excretion $>12 \%$, and (VII) correction of hyponatremia through fluid restriction.

Plasma levels of chlorine, sodium, potassium, creatinine, glucose, hemoglobin ( $\mathrm{Hb})$, hematocrit, urea, bicarbonate, alanine transaminase (ALT) and aspartate transaminase (AST) were assayed using the Roche Cobas 8000 automatic biochemical analyzer (Roche Diagnostics, Basel, Switzerland). Plasma NT-proBNP concentration was determined by an immunoelectrochemiluminescence method on Roche Cobas 8000/E602 immunoassay module. The osmolality of plasma and urine was measured using the electrode method. Vivid E9 ultrasound scanner (GE Vingmed, Horten, Norway) was used to measure the left ventricular ejection fraction (LVEF, \%).

\section{Statistical analysis}

The normality of data distribution was tested by Kolmogorov-Smirnov test. Variables with skewed distribution were expressed as median and quartile ranges (25 th to 75 th percentiles). Continuous variables with normal distribution were expressed as mean \pm standard deviation
(SD). Categorical variables were expressed as numbers and percentages. Variables with normal distribution were compared between two groups with the unpaired Student's $t$ test. Difference of NT-proBNP levels and blood pressure levels between two groups was analyzed by Mann-Whitney $\mathrm{U}$ test. Difference of NT-proBNaP levels among three groups was compared by Kruskal-Wallis $\mathrm{H}$ test, and then Bonferroni pairwise comparisons were performed if $\mathrm{P}<0.05$. Chi-square test was used to compare categorical variables. Data were analyzed by software SPSS version 16.0. Difference was considered to be significant when $\mathrm{P}<0.05$ (two-tailed).

\section{Results}

The study performed strict exclusion and inclusion criteria according to the aforementioned methods. A PRISMA flowchart is shown in Figure 1. A total of 56 patients who had adequate clinical information were involved in this study (Table 1). Patient ages in the hyponatremic group were markedly older compared to the eunatremic group $(55.96 \pm 14.40$ vs. $39.47 \pm 14.67$ years). The baseline blood pressure levels were significantly higher in the hyponatremic group (systolic blood pressure: median $163 \mathrm{mmHg}$, IQR, 115-176 mmHg vs. median $127 \mathrm{mmHg}$, IQR, 102-149 $\mathrm{mmHg}, \mathrm{P}<0.05$; diastolic blood pressure: median $112 \mathrm{mmHg}$, IQR, 84-131 mmHg vs. median $86 \mathrm{mmHg}$, IQR, 63-104 mmHg, $\mathrm{P}<0.05$ ), with more patients treated with antihypertensives compared to the eunatremic group $(60.6 \%$ vs. $34.8 \%, \mathrm{P}<0.05)$. Similar LVEF, AST and eGFR (evaluated glomerular filtration rate) levels were observed between two groups. Patients with hyponatremia had less $\mathrm{Hb}$ and lower hematocrit $(\mathrm{P}<0.05)$, likely reflecting diluted hyponatremia. Meanwhile, hyponatremic patients had significantly higher NT-proBNP levels. Nevertheless, no direct correlation was found between sodium and NT-proBNP levels in hyponatremic patients $(\mathrm{r}=0.093, \mathrm{P}=0.607)$.

Finally, five patients had died in the SIADH group, while all patients in eunatremic group had recovered. To further explore the relationship between NT-proBNP levels and sodium levels, NT-proBNP levels were compared between hyponatremia phase and eunatremia phase. Although there was downward trend of NT-proBNP levels after the correction of hyponatremia in disease remission phase (median $419.5 \mathrm{pg} / \mathrm{mL}$, IQR, 163.0-581.5 pg/mL) vs. median $212.0 \mathrm{pg} / \mathrm{mL}$, IQR, 123.0-298.5 pg/mL), the difference was not statistically significant $(\mathrm{P}=0.130$, Figure 2$)$. 


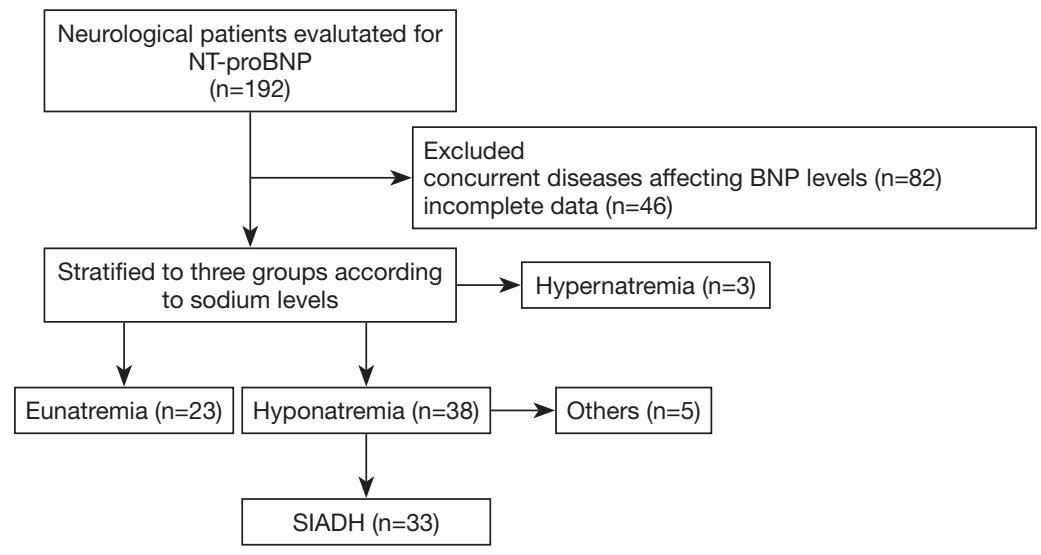

Figure 1 PRISMA flowchart of the study.

Table 1 Baseline characteristics of hyponatremic and eunatremic patients

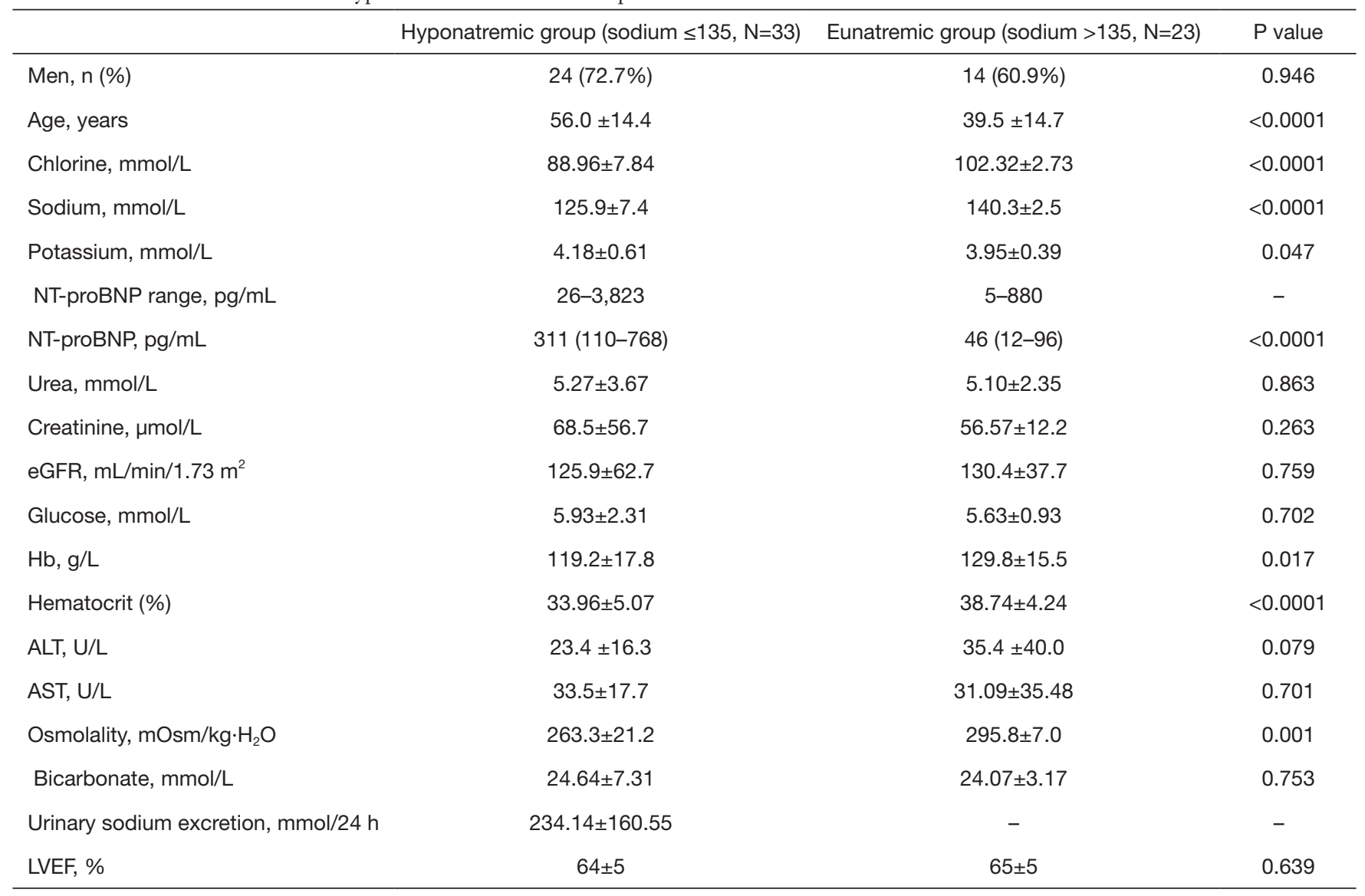

Data are expressed as mean \pm SD for data with normal distribution, or median (IQR) for data with skewed distribution, or number (percentage) for categorical data. $\mathrm{P}$ values less than 0.05 are marked in bold and were considered to be statistically significant. Difference of continuous variables was compared using unpaired Student's $t$ test unless otherwise indicated. Analysis was performed using MannWhitney $U$ test. eGFR, estimated glomerular filtration rate; Hb, hemoglobin; ALT, alanine transaminase; AST, aspartate transaminase; LVEF, left ventricular ejection fraction. 
The concurrent complications and disease severities varied in these SIADH patients. These patients were divided into three groups according to the number of severe complications. These complications were defined as acute respiratory failure, pneumonia, acute hepatic dysfunction, hemorrhage of upper digestive tract, anemia $(\mathrm{Hb}<90 \mathrm{~g} / \mathrm{L})$ and coma. No significant difference of serum sodium levels was observed among three groups $(\mathrm{P}=0.31)$. Patients with two or more complications (group $\mathrm{C}$ ) had higher levels of NT-proBNP than patients without complication (group A) $(\mathrm{P}=0.03)$. It seems that influence of disease severities on NT-proBNP levels was more obvious than serum sodium concentration (Table 2).

\section{Discussion}

Hyponatremia commonly occurs in patients with neurological diseases. The main cause of neurological hyponatremia has been attributed to SIADH. Secondary

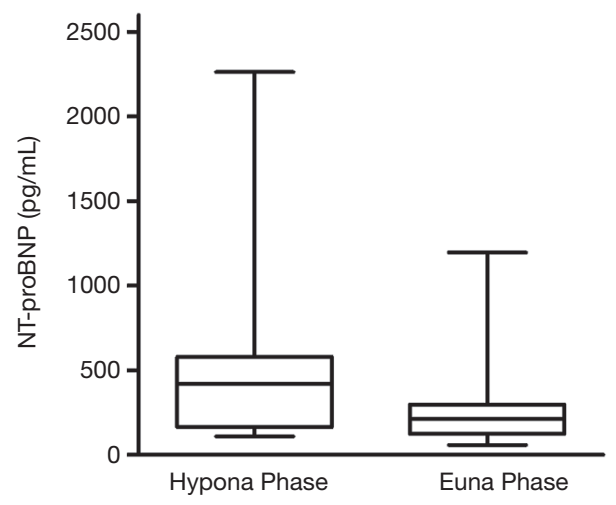

Figure 2 Comparison of mean NT-proBNP levels before and after correction of hyponatremia in patients with SIADH. Hypona, hyponatremia; Euna, eunatremia; NT-proBNP, N-terminal probrain natriuretic peptide; SIADH, syndrome of inappropriate antidiuretic hormone secretion. to cerebral injuries, inappropriate secretion of $\mathrm{ADH}$ in hypothalamus leads to water retention and diluted hyponatremia. The abnormal release of $\mathrm{ADH}$ has been proposed to be caused by several reasons. Firstly, intracranial hemorrhage, infection or injury can interrupt the neuronal crosstalk or hormonal feedback mechanisms (25). Secondly, ADH secretion can be triggered by stress, hypovolemic state and increased ICP (26). The ECV state of SIADH is hypervolemic or euvolemic.

BNP is mainly synthesized by ventricular cardiomyocytes triggered by ventricular stretch and volume overload. Elimination pathways of BNP in plasma include glomerular infiltration, natriuretic receptors and neutral endopeptidases. In contrast, elimination of NTproBNP largely depends on glomerular infiltration (27). Therefore, BNP and NT-proBNP have been extensively utilized as biomarkers in acute or chronic congestive heart failure. In addition to heart failure and glomerular infiltration, plasma BNP levels are influenced by several other confounding factors, including age, gender, body mass index, inflammation, steroid agents, volume state, neurological insults, stress, arrhythmia, acute respiratory distress syndrome and so on (28-32). Among these factors, it seems that higher NT-proBNP levels always parallel with a disease or aging state. Except for the influence of gender, steroid agents and body mass index (32), other factors, such as age, inflammation, volume state, neurological insults, stress, arrhythmia and acute respiratory distress syndrome all positively correlated with higher NT-proBNP levels. So, elevated NT-proBNP levels are frequently associated with worse prognosis in various neurological diseases, such as acute ischemic stroke, traumatic brain injury and SAH $(22,23,33)$. It has long been disputed whether NTproBNP serves as a biomarker for volume state or for overall disease severities. Our study doesn't support the notion that NT-proBNP levels are reliable predictor for volume state, for plasma NT-proBNP levels were notably raised in patients with SIADH compared to eunatremic

Table 2 The effect of the amount of complications on serum sodium and NT-proBNP levels in SIADH patients

\begin{tabular}{lcccc}
\hline Group & Number of complications & Cases & Sodium range $(\mathrm{mmol} / \mathrm{L})$ & NT-proBNP $\mathrm{range}(\mathrm{pg} / \mathrm{mL})$ \\
\hline A & 0 & 7 & $112.7-133.5$ & $42-419^{*}$ \\
B & 1 & 6 & $105.9-131.6$ & $53-2,576$ \\
C & $\geq 2$ & 20 & $115.8-134.6$ & $26-3,823$ \\
\hline
\end{tabular}

Group A compared with group $B, P=0.565$; *, group A compared with group $C, P=0.03$; group $B$ compared with group $C$, $P=0.36$. NTproBNP, N-terminal pro-brain natriuretic peptide; SIADH, syndrome of inappropriate antidiuretic hormone secretion. 
patients. We diagnosed these patients as SIADH according to widely accepted standards published previously (24). Cerebral salt wasting syndrome (CSWS) was excluded in these patients by evaluating volume state and treatment responsiveness of fluid restriction. In our study, extracellular volume seems to be slightly expanded in SIADH patients, manifesting as lower hematocrit compared to eunatremic patients. Similarly, positive fluid balance was generally observed in SIADH patients with SAH (34). Studies from volunteers have shown that plasma BNP levels appear to be insensitive to volume expansion or increased sodium uptake $(35,36)$. It has been showed that high BNP levels are related to occurrence of severe hypovolemia and possibly hyponatremia in SAH patients, but data do not support a role for BNP measurement to differentiate between hypovolemic and non-hypovolemic hyponatremia (37). Hence, it seems that BNP or NT-proBNP levels were not reliable biomarkers to evaluate volume status in pathophysiological settings.

Both systematic and local inflammation may contribute to increased secretion of AVP. Several inflammatory factors, such as prostaglandin-E2, interleukin (IL)-1 $\beta$, IL-6 and tumor necrosis factor- $\alpha$, have been confirmed to be induced in hypothalamus and pituitary after challenge with lipopolysaccharides in animal models (38). In addition to cerebral infection, sterile inflammation caused by damage-associated molecular patterns, such as ischemia, hemorrhage and trauma in the brain, also contributes to markedly increased production of IL- $1 \beta$ in glia cells (39). These factors produced locally or possibly systematically directly stimulate the synthesis and secretion of AVP in neuroendocrine cells within paraventricular (PVN) and supraoptic (SON) nuclei. Aberrant secretion of AVP reflects the severity of inflammation and stress response in the hypothalamus. It has been demonstrated in primate models that BNP mainly exists in PVN and SON where large numbers of AVP-positive neurons exist in the hypothalamus (20). BNP levels increased shortly after traumatic brain injury or stroke, irrespective of the existence of cardiac dysfunction or not $(33,40)$. It raises the possibility that inflammatory insults and stress response might affect secretion of BNP from the hypothalamus. However, direct evidence to support this notion is lacking.

The fluctuation of NT-proBNP levels during stress responses varied from person to person. One underlying mechanism to explain is the difference of cardiopulmonary performance among different persons. Athletes who had potent cardiopulmonary fitness tended to have lower NTproBNP levels both at rest and during physical stress than age-matched untrained healthy volunteers (41). In our study, SIADH patients had relatively elder ages and presumably worsened cardiopulmonary fitness. Thus, they tended to have elevated NT-proBNP levels under stress responses. The magnitude of stress responses may also contribute to promotion of secretion of AVP and NT-proBNP, for patients with more complications had significantly elevated NT-proBNP levels. Based on these reasons, we postulate from our study that NT-proBNP levels serve as predictor for disease severity while not volume status in pathophysiological settings, at least in patients with neurological diseases.

Nevertheless, higher NT-proBNP levels seem to be not consistently associated with hyponatremia (37). No difference of NT-proBNP had been found during and after the episode of hyponatremia in patients with SAH (1). In patients who underwent neurosurgery, NT-proBNP levels in SIADH patients were significantly lower than those of CSWS patients (42). These discrepancies from different studies may be attributed to primary neurological diseases, complications and disease severities.

Our study exists several limitations. Firstly, the sample size is small and the causes of primary diseases are heterogeneous. One reason for the small sample size is that we strictly screened SIADH patients and excluded patients with severe kidney failure and heart failure. Although these patients suffered from neurological diseases, etiological causes varied among these patients. Secondly, the retrospective study was cross-sectional. NT-proBNP measurements were obtained after onset of hyponatremia, while baseline levels of NT-proBNP before disease onset were lacking. Co-existing factors are complicated to influence plasma BNP levels. Therefore, a causal relationship between NT-proBNP levels and SIADH remains unclear. However, higher NT-proBNP levels always indicate worse conditions, which is the same as SIADH. In our study, as the patients with SIADH progressed into remission phase, there was a downward trend in NTproBNP levels compared to the hyponatremic phase. This alteration could be considered as a self-controlled study to maximally eliminate the influence of co-existing factors. Thirdly, the cross-sectional study enrolled patients who sequentially hospitalized in a time window. Selection bias unavoidably affected the analysis. We suggest that further study be designed with well-controlled comparison. 


\section{Conclusions}

We conclude that neurological patients with SIADH have higher plasma NT-proBNP levels and poorer clinical prognosis than those without. NT-proBNP possibly serves as disease severity biomarker while not extracellular volume status in critically ill neurological patients.

\section{Acknowledgments}

We are grateful to the colleagues in Tongji Hospital affiliated to Tongji Medical College, Huazhong University of Science and Technology for their collaboration on the study. We also express our gratitude to the patients and their family members.

Funding: This research was supported by National Natural Science Foundation of China (Grant Number 81770817).

\section{Footnote}

Reporting Checklist: The authors have completed the STROBE reporting checklist. Available at http://dx.doi. org/10.21037/atm-20-3413

Data Sharing Statement: Available at http://dx.doi. org/10.21037/atm-20-3413

Conflicts of Interest: All authors have completed the ICMJE uniform disclosure form (available at http://dx.doi. org/10.21037/atm-20-3413). The authors have no conflicts of interest to declare.

Ethical Statement: The authors are accountable for all aspects of the work in ensuing that questions related to the accuracy or integrity of any part of the work are appropriately investigated and resolved. All procedures were carried out in accordance with the principles of the Declaration of Helsinki (as revised in 2013). This study was approved by the ethics committee of Tongji Hospital, Tongji Medical College, Huazhong University of Science and Technology (Institutional Review Board Approval No. TJ-C20190101). Written informed consent was obtained from all participating patients on admission.

Open Access Statement: This is an Open Access article distributed in accordance with the Creative Commons Attribution-NonCommercial-NoDerivs 4.0 International License (CC BY-NC-ND 4.0), which permits the non- commercial replication and distribution of the article with the strict proviso that no changes or edits are made and the original work is properly cited (including links to both the formal publication through the relevant DOI and the license). See: https://creativecommons.org/licenses/by-nc-nd/4.0/.

\section{References}

1. Hannon MJ, Finucane FM, Sherlock M, et al. Clinical review: Disorders of water homeostasis in neurosurgical patients. J Clin Endocrinol Metab 2012;97:1423-33.

2. Peri A. Management of hyponatremia: causes, clinical aspects, differential diagnosis and treatment. Expert Rev Endocrinol Metab 2019;14:13-21.

3. Hao J, Li Y, Zhang X, et al. The prevalence and mortality of hyponatremia is seriously underestimated in Chinese general medical patients: an observational retrospective study. BMC Nephrol 2017;18:328.

4. Waikar SS, Mount DB, Curhan GC. Mortality after hospitalization with mild, moderate, and severe hyponatremia. Am J Med 2009;122:857-65.

5. Hawkins RC. Age and gender as risk factors for hyponatremia and hypernatremia. Clin Chim Acta 2003;337:169-72.

6. Zheng Y, Zheng FP, Li H. The prevalence and causes of hyponatremia in hospitalized patients. Zhonghua Nei Ke Za Zhi 2020;59:29-34.

7. Kutz A, Ebrahimi F, Sailer CO, et al. Seasonality of Hypoosmolar Hyponatremia in Medical Inpatients Data from a Nationwide Cohort Study. J Clin Endocrinol Metab 2020;105:dgz320.

8. Sherlock M, O'Sullivan E, Agha A, et al. Incidence and pathophysiology of severe hyponatraemia in neurosurgical patients. Postgrad Med J 2009;85:171-5.

9. Lim AKH, Paramaswaran S, Jellie LJ, et al. A CrossSectional Study of Hyponatremia Associated with Acute Central Nervous System Infections. J Clin Med 2019;8:1801.

10. Fraser JF, Stieg PE. Hyponatremia in the neurosurgical patient: epidemiology, pathophysiology, diagnosis, and management. Neurosurgery 2006;59:222-9; discussion 222-9.

11. Cui H, He G, Yang S, et al. Inappropriate Antidiuretic Hormone Secretion and Cerebral Salt-Wasting Syndromes in Neurological Patients. Front Neurosci 2019;13:1170.

12. Chawla A, Sterns RH, Nigwekar SU, et al. Mortality and serum sodium: do patients die from or with hyponatremia? Clin J Am Soc Nephrol 2011;6:960-5. 
13. Schrier RW, Sharma S, Shchekochikhin D.

Hyponatraemia: more than just a marker of disease severity? Nat Rev Nephrol 2013;9:37-50.

14. Basu A, Ryder RE. The syndrome of inappropriate antidiuresis is associated with excess long-term mortality: a retrospective cohort analyses. J Clin Pathol 2014;67:802-6.

15. Potter LR. Natriuretic peptide metabolism, clearance and degradation. FEBS J 2011;278:1808-17.

16. Pagel-Langenickel I. Evolving Role of Natriuretic Peptides from Diagnostic Tool to Therapeutic Modality. Adv Exp Med Biol 2018;1067:109-31.

17. La Villa G, Stefani L, Lazzeri C, et al. Acute effects of physiological increments of brain natriuretic peptide in humans. Hypertension 1995;26:628-33.

18. Kakoullis L, Giannopoulou E, Papachristodoulou E, et al. The utility of brain natriuretic peptides in septic shock as markers for mortality and cardiac dysfunction: A systematic review. Int J Clin Pract 2019;73:e13374.

19. Sudoh T, Kangawa K, Minamino N, et al. A new natriuretic peptide in porcine brain. Nature 1988;332:78-81.

20. Abdelalim EM, Takada T, Torii R, et al. Molecular cloning of BNP from heart and its immunohistochemical localization in the hypothalamus of monkey. Peptides 2006;27:1886-93.

21. García-Berrocoso T, Giralt D, Bustamante A, et al. B-type natriuretic peptides and mortality after stroke: a systematic review and meta-analysis. Neurology 2013;81:1976-85.

22. Zhao YH, Gao H, Pan ZY, et al. Prognostic Value of NTproBNP After Ischemic Stroke: A Systematic Review and Meta-analysis of Prospective Cohort Studies. J Stroke Cerebrovasc Dis 2020;29:104659.

23. Nyberg C, Karlsson T, Ronne-Engstrom E. Predictors of increased cumulative serum levels of the N-terminal prohormone of brain natriuretic peptide 4 days after acute spontaneous subarachnoid hemorrhage. J Neurosurg 2014;120:599-604.

24. Spasovski G, Vanholder R, Allolio B, et al. Clinical practice guideline on diagnosis and treatment of hyponatraemia. Nephrol Dial Transplant 2014;29 Suppl 2:11-39.

25. Albanese A, Hindmarsh P, Stanhope R. Management of hyponatraemia in patients with acute cerebral insults. Arch Dis Child 2001;85:246-51.

26. Cole CD, Gottfried ON, Liu JK, et al. Hyponatremia in the neurosurgical patient: diagnosis and management. Neurosurg Focus 2004;16:E9.

27. Hall C. NT-ProBNP: the mechanism behind the marker. J Card Fail 2005;11:S81-3.
28. Baba O, Izuhara M, Kadota S, et al. Determinant factors of plasma B-type natriuretic peptide levels in patients with persistent nonvalvular atrial fibrillation and preserved left ventricular systolic function. J Cardiol 2009;54:402-8.

29. Felker GM, Petersen JW, Mark DB. Natriuretic peptides in the diagnosis and management of heart failure. CMAJ 2006;175:611-7.

30. Moraes JC, Ribeiro AC, Saad CG, et al. NT-proBNP levels may be influenced by inflammation in active ankylosing spondylitis receiving TNF blockers: a pilot study. Clin Rheumatol 2013;32:879-83.

31. Stolz D, Breidthardt T, Christ-Crain M, et al. Use of B-type natriuretic peptide in the risk stratification of acute exacerbations of COPD. Chest 2008;133:1088-94.

32. Madamanchi C, Alhosaini H, Sumida A, et al. Obesity and natriuretic peptides, BNP and NT-proBNP: mechanisms and diagnostic implications for heart failure. Int J Cardiol 2014;176:611-7.

33. Wu X, Sha H, Sun Y, et al. N-terminal pro-B-type natriuretic peptide in patients with isolated traumatic brain injury: a prospective cohort study. J Trauma 2011;71:8205; discussion 825 .

34. Hannon MJ, Behan LA, O'Brien MM, et al. Hyponatremia following mild/moderate subarachnoid hemorrhage is due to SIAD and glucocorticoid deficiency and not cerebral salt wasting. J Clin Endocrinol Metab 2014;99:291-8.

35. Wambach G, Koch J. BNP plasma levels during acute volume expansion and chronic sodium loading in normal men. Clin Exp Hypertens 1995;17:619-29.

36. Ishimitsu T, Minami J, Nishikimi T, et al. Responses of natriuretic peptides to acute and chronic salt loading in normotensive and hypertensive subjects. Hypertens Res 1998;21:15-22.

37. Dorhout Mees SM, Hoff RG, Rinkel GJ, et al. Brain natriuretic peptide concentrations after aneurysmal subarachnoid hemorrhage: relationship with hypovolemia and hyponatremia. Neurocrit Care 2011;14:176-81.

38. Gatti S, Bartfai T. Induction of tumor necrosis factor-alpha mRNA in the brain after peripheral endotoxin treatment: comparison with interleukin-1 family and interleukin-6. Brain Res 1993;624:291-4.

39. Brough D, Rothwell NJ, Allan SM. Interleukin-1 as a pharmacological target in acute brain injury. Exp Physiol 2015;100:1488-94.

40. Salem R, Vallee F, Depret F, et al. Subarachnoid hemorrhage induces an early and reversible cardiac injury associated with catecholamine release: one-week follow-up study. Crit Care 2014;18:558. 
41. Popovic D, Popovic B, Plecas-Solarovic B, et al. The interface of hypothalamic-pituitary-adrenocortical axis and circulating brain natriuretic peptide in prediction of cardiopulmonary performance during physical stress. Peptides 2013;47:85-93.

Cite this article as: Zeng QL, He WT, Yuan G. Higher plasma NT-proBNP levels correlate with syndrome of inappropriate antidiuretic hormone and poor prognosis in neurological patients. Ann Transl Med 2021;9(1):28. doi: 10.21037/atm-20-3413
42. Tobin G, Chacko AG, Simon R. Evaluation of NT-

ProBNP as a marker of the volume status of neurosurgical patients developing hyponatremia and natriuresis: A pilot study. Neurol India 2018;66:1383-8. 